\title{
New Progress in Job Design Theory: Literature Review of Job Crafting
}

\author{
Xiao Yuchun ${ }^{1, a}$, Ke Junqun ${ }^{2, b, *}$ \\ ${ }^{1}$ School of Business Administration, Zhejiang Gongshang University, Hangzhou Zhejiang, China \\ ${ }^{2}$ School of Business Administration, Zhejiang Gongshang University, Hangzhou Zhejiang, China \\ axiaoyuchun@126.com \\ b1531936844@qq.com
}

\begin{abstract}
Job design is an important part of organizational research and the key to the sustainable development of an organization. Job crafting is the development and innovation of the traditional work design theory, which subverts the traditional top-down work design method and aims to stimulate the enthusiasm of employees and improve their work efficiency in a bottom-up way. In this paper, based on the recent progress of job crafting research at home and abroad, the job crafting is sorted out and supplemented to provide insights for follow-up research.
\end{abstract}

Keywords: job crafting, job design, organization manage

\section{工作设计理论研究新进展：工作重塑研究综述}

\author{
肖余春 $1, \mathrm{a}$ 柯俊群 $2, \mathrm{~b}^{*}$ \\ ${ }^{\prime}$ 浙江工商大学工商管理学院, 杭州, 浙江, 中国 \\ ${ }^{2}$ 浙江工商大学工商管理学院, 杭州, 浙江, 中国 \\ ${ }^{a}$ xiaoyuchun@126. com \\ ${ }^{b} 1531936844 @ q q . c o m$

\section{摘要} \\ 工作设计是组织研究的重要一环, 也是组织持续发展的关键。工作重塑是传统工作设计理论的发展和 \\ 创新, 颠覆了传统自上而下的工作设计方式, 旨在以一种自下而上的工作方式来激发员工工作积极性, \\ 提高员工工作效率, 从而为组织带来利益。本文基于近几年国内外有关工作重塑研究的新进展, 对工 \\ 作重塑理论进行梳理和补充, 为后续研究 提供见解。
}

关键词: 工作重塑, 工作设计, 组织管理

\section{1. 工作重塑的概念和研究进展}

传统的工作设计视角主要关注的是员工如何理解 工作环境中的客观任务特征和社会信息, 从而产生对工 作的态度和动机反应 ${ }^{[1]}$, 是一种自上而下的由组织和管 理者发起的工作设计方式, 旨在激励员工的工作积极性。 但是这种工作设计方式往往低估了员工在积极塑造构 成工作的任务和社会关系方面所发挥的作用。即使在最 受限制和最常规的工作中, 员工也能对工作的本质产生 一些影响。在此基础上, Wrzesniewski and Dutton (2001) ${ }^{[2]}$ 提出了 “工作重塑” 这个概念, 并将其 定义为 “个体在工作任务或工作关系边界中所做出的身 体和认知上的改变”。并将工作重塑分为任务重塑、关
系重塑和认知重塑。任务重塑包括通过改变工作中完成 的工作任务的数量、范围或类型来改变工作的任务边界。 例如, 医院的清洁工在完成本职工作之外还会帮助医护 人员, 或是帮助患者。关系重塑是指通过改变工作中与 他人互动的方式来参与人际关系的构建。例如, 计算机 技术人员将为同事提供帮助作为与他人建立联系的一 种方式。认知重塑主要指改变对工作看法和态度。例如, 图书管理员将工作看成是有益于人类知识传播、促进社 会进步的崇高工作，而不仅仅是分类、记账、上柜和上 架等机械式劳动。之后, Wrzesniewski 等 $(2012)^{[3]}$ 扩 展了工作重塑的维度划分, 进一步提出了技能重塑, 代 表了员工在工作中自发的努力, 以改变他们的技能, 更 好地开展自己的工作。

基于工作需求 - 工作资源理 论 ${ }^{[4]}$ (Bakker \& 
Demerouti, 2007), Tims and Bakker (2010) ${ }^{[5]}$ 将工作重 塑定义为 “员工可能做出的改变, 以平衡他们的工作需 求和工作资源与他们的个人能力和需求” 。工作需求指 的是需要持续的体力、情感或脑力劳动的工作方面, 而 工作资源指的是能够刺激个人成长和发展、减少工作需 求或对实现工作目标有作用的工作方面 ${ }^{[4]}$ 。具体来说, Tims 等 (2012) ${ }^{[5]}$ 确定了工作重塑的四个不同维度：（1） 增加结构性工作资源（例如，增加个人的发展机会）;

(2) 增加社会工作资源 (如向上司寻求反馈); (3) 增加挑 战性的工作要求 (例如, 承担额外的任务); (4) 减少阻 碍工作的要求 (例如, 确保工作在情感上不那么紧张)。

Wrzesniewski and Dutton(2001) ${ }^{[2]}$ 关注任务/关系 /认知边界的变化, 而 Tims 等 (2012) ${ }^{[5]}$ 关注工作特征 的变化。Wrzesniewski and Dutton (2001) ${ }^{[2]}$ 认为重塑 是提高工作意义和工作身份的一种方式, 而 Tims 等 (2012) ${ }^{[5]}$ 认为重塑是平衡工作资源和需求的一种方式, 从而实现人与工作的契合。之后关于工作重塑的后续研 究也是基于这两种不同的视角, 因此, 为了将两种观点 进行综合研究，学者们也做出了不少的努力。

基于角色-资源两个视角以及接近-回避两种形式 对工作重塑进行的整合, Bruning and Campion (2018) ${ }^{[6]}$ 提出了角色-资源-接近-回避工作重塑模型, 包含四个 类别: 接近角色重塑、接近资源重塑、回避角色重塑和 回避资源重塑; 并将工作重塑定义为员工为改善自己的 工作而对工作进行的改变。这些变化可以采取结构、社 会和认知的形式。角色视角下的工作重塑主要是根据个 人的工作角色以及他们对任务、关系边界的改变以及认 知领域进行定义 ${ }^{[7,8]}$ 。资源视角下的工作重塑主要借鉴 的是工作需求一资源模型, 认为工作重塑的目的在于寻 求资源和回避需求 ${ }^{[5,9]}$ 。

虽然 Bruning and Campion (2018) ${ }^{[6]}$ 对整合两种 视角的工作重塑做出了重要的贡献, 但是他们的研究也 存在局限性 ${ }^{[10]}$ 。Zhang and Parker (2018) 认为角色和 资源视角都涉及到动机元素, 因此他们提出了工作重塑 的三级层次结构: 结构的第一个层次是将工作重塑分为 前进和回避导向的工作重塑。第二层次是围绕认知重塑 的争论, 将重塑形式分为认知重塑和行为重塑。最后一 个层次在于将工作重塑内容分为资源重塑和需求重塑。 基于三个层次结构, 将工作重塑分为八种类型: 前进资 源重塑 (行为)、前进需求重塑 (行为)、前进资源重塑 (认知)、前进需求重塑 (认知)、回避资源重塑 (行为)、 回避需求重塑 (行为)、回避资源重塑 (认知)、回避资 源重塑 (认知)。作者认为, 前进重塑在于寻求工作的积 极方面, 而回避重塑在于避免和逃避工作的消极方面, 可能会导致消极结果的发生 ${ }^{[10]}$ 。但是作者也仅仅是进行 理论上的探讨, 并没有对其进行实证验证。

基于资源和角色视角, Lichtenthaler and Fischbach (2019) ${ }^{[11]}$ 对工作重塑进行的元分析总结 ${ }^{[11]}$ 。 基于调节焦点理论, 区分了促进导向的工作重塑 (增加 工作资源和挑战性工作需求, 面向扩展形式的任务、关 系和认知重塑) 和预防导向的工作重塑 (减少阻碍性工 作需求; 面向限制形式的任务、关系和认知重塑)。基于 调节焦点理论和工作需求-资源理论, 进一步推理促进
导向的工作重塑与动机、健康和绩效正相关，因为促进 导向的工作重塑在有形的工作角色边界上产生有利的 变化（例如, 激发工作特征的增加）和无形工作角色感 知的有利变化（例如, 积极情绪的增加） ${ }^{[6,12,13]}$ 。预防导 向的工作重塑与动机、健康和绩效负相关, 因为预防导 向的工作重塑不会在有形的工作角色边界中产生有利 的变化 (例如, 在繁重的工作特征上没有变化), 而在工 作角色感知上却产生了不利的变化 (例如, 负面情绪的 增加 $)^{[6,12,13]}$ 。但是, 作者也只是在整合现有研究基础上 进行的推理, 因此, 需要进一步实证分析促进导向和预 防导向的工作重塑对工作结果的影响。

基于个人需求和调节焦点理论，Bind1, Unsworth, Gibson and Unsworth (2019) ${ }^{[14]}$ 也区分了促进和预防两 种形式的工作重塑。根据调节焦点理论, 个人可以选择 以探索的方式来扩展工作和关系边界, 以最大限度地增 加个人收益, 即进行促进导向的工作重塑; 或者是注重 安全和减少负面结果的发生而缩小工作和关系边界, 即 进行预防导向的工作重塑。虽然已有研究已经提到的工 作重塑的扩展和限制形式，但学术界大多关注的是促进 形式的工作重塑, 而限制性或预防性的工作重塑主要被 定位为否定的形式。相比之下, Bind1, Unsworth, Gibson and Unsworth (2019) 认为以预防为导向的工作 重塑也代表了对工作的主动性改变, 可以防止负面结果 的发生。预防导向的工作重塑不同于工作中的退缩行为, 它仍然构成一种主动性行为的形式。

注重促进的个人倾向于将目标视为希望和抱负, 并 在不因疏忽或缺乏成就而犯错误的情况下努力取得成 果 ${ }^{[15]}$ 。促进导向的工作重塑代表着一种 “收益” 的方式, 员工可以通过这种方式来增加和扩展现有的工作方面。

综上所述, 目前关于促进型和预防型工作重塑的实 证研究较少, 且预防型工作重塑对结果变量的作用效果 还处在探索阶段。因此, 本研究基于 Bind1, Unsworth, Gibson and Unsworth (2019) 对工作重塑的划分, 进一 步对促进型和预防型工作重塑进行实证分析研究, 为后 续研究提供思路和见解, 促进后续学者对两种形式工作 重塑的深层次分析。

\section{2. 工作重塑的测量}

目前, 关于工作重塑的测量研究较为丰富, 许多学 者开发了多种测量工具。一方面, Wrzesniewski and $\operatorname{Dutton}(2001)$ 根据调研与访谈首次提出工作重塑, 并将 其划分为任务重塑、关系重塑和认知重塑, 但是没有设 计出具体的量表 ${ }^{[2]}$ 。Slemp and Vella-Brodrick (2014) 在前人研究的基础上, 开发了 15 题项量表, 包括任务、 关系、认知重塑三个方面。该量表经过诸多国内外学者 的研究检验证实, 具有较高的信效度 ${ }^{[16]}$ 。另一方面, 根 据工作要求一一资源模型, Tims, Bakker and Derks (2012) 划分了工作重塑的四个维度。该研究在保 证较大样本量的基础上, 经因子分析保留了 21 个题项, 信效度较好, 认可度较高, 应用较为广泛 ${ }^{[9]}$ 。

在关于经两种视角整合的研究中, Bruning and 
的影响因素之一。职位等级相对较低的员工更容易适应 工作环境并创造更多的工作重塑机会, 职级等级相对较 高的员工反而会在工作重塑过程中感受到更多的限制, 尽管他们拥有更多的正式权力和自主权。

工作重塑作为一种自下而上的工作设计方式，对个 人和组织都会带来积极的影响。员工根据自身能力和偏 好对工作进行重塑, 能够提高员工的工作满意度和工作 幸福感, 对员工的情绪和心理都能产生较好的影响, 员 工会更加投入自身的工作，促使个人绩效得到提升，从 而能够提升组织绩效, 为组织带来更大的利益。研究表 明, 工作重塑对个人影响不仅表现在员工个人体验和感 知方面, 还会对员工发家庭生活产生影响。后续相关研 究发现, 员工工作重塑的直接目的在与通过改变工作边 界事工作与自身能力偏好相匹配, 但在客观上能促进工 作流和技术创新, 提高员工个人和组织生产效率, 使组 织受益 ${ }^{[18]}$ 。

\section{4. 结束语}

员工的工作重塑作为一种主动性行为, 能够为个人 和组织带来好处。现有关于工作重塑的研究也多集中于 工作重塑发积极方面, 并且, 学者也呼吁组织应该重视 个人的主观能动性, 要为员工创造相对宽松的氛围, 鼓 励员工在应对工作环境变化时主动做出调整, 为企业带 来活力。但是, 工作重塑作为一种主动性行为或行为策 略, 也能为组织带来不利的影响, 当个人利益与组织利 益向冲突时, 个体很可能做出不利于组织的自我改变行 为。此外, 目前学术界对工作重塑的消极影响研究相对 空白, 后续研究可从这方面入手, 丰富工作重塑理论。

\section{References}

[1] Borowsky, B. R., Mcmahan, W. C., Griffin, W. A., Norris, F. H., \& Ruffolo, R. R. (1980). Computerized graphic methods for determining dissociation constants of agonists, partial agonists, and competitive antagonists in isolated smooth muscle preparations., 4(2), 165-178.

[2] Wrzesniewski, A., \& Dutton, J., E. (2001). Crafting a job: revisioning employees as active crafters of their work. The Academy of Manage ment Review, 26(2), 179-201.

[3] Wrzesniewski, A., Berg, J. M., Grant, A. M., Kurkoski, J., \& Welle, B.(2012). Job crafting in motion: Achieving sustainable gains in happiness and performance. Paper presented at the Annual Meeting of the Academy of Management, Boston, MA.

[4] Bakker, A.B., Demerouti, E. (2007). Job resources boost work engagement, particularly when job demands are high. Journal of Educational Psychology, 99(2), 274284. 
[5] Tims, M., \& Bakker, A. B. (2010). Job crafting: towards a new model of individual job redesign. SA Journal of Industrial Psychology, 36(2).

[6] Bruning, P. F., Campion, M. A. (2018). A roleresource approach-avoidance model of job crafting: A multi-method integration and extension of job crafting theory. Academy of Management Journal,61(2), 499522.

[7] Berg, J. M., Wrzesniewski, A., \& Dutton, J. E. (2010b). Perceiving and responding to challenges in job crafting at different ranks: When proactivity requires adaptivity. Journal of Organizational Behavior, 31: 158186.

[8] Lu, C. Q., Wang, H. J., Lu, J. J., Du, D. Y., \& Bakker, A. B. (2014). Does work engagement increase personjob fit? the role of job crafting and job insecurity. Journal of Vocational Behavior, 84(2), 142-152.

[9] Bakker, A. B., Tims, M., \& Derks, D. (2012). Proactive personality and job performance: the role of job crafting and work engagement. Human Relations, 65(10), 1359-1378.

[10] Zhang, F., \& Parker, S. K. (2018). Reorienting job crafting research: a hierarchical structure of job crafting concepts and integrative review. Journal of Organizational Behavior.

[11] Lichtenthaler, P. W., \& Fischbach, A. (2018). A meta-analysis on promotion- and prevention-focused job crafting. European Journal of Work and Organizational Psychology, 1-21.

[12] Lichtenthaler, P. W., \& Fischbach, A. (2016). Job crafting and motivation to continue working beyond retirement age. Career Development International, 21(5), 477-497.

[13] Tims, M., Bakker, A. B., \& Derks, D. (2013). The impact of job crafting on job demands, job resources, and well-being. Journal of Occupational Health Psychology, $18(2), 230-40$.

[14] Bindl, U,. K., Unsworth, K., L., Gibson, C., B., \& Stride, C, B.(2019). Job Crafting Revisited: Implications of an Extended Framework for Active Changes at Work. Journal of Applied Psychology, 104(5),605-628.

[15] Ellen Crowe, \& E.Tory Higgins.(1997). Regulatory focus and strategic inclinations: promotion and prevention in decision-making, 69(2), 117-132.

[16] Slemp, G. R., \& Vella-Brodrick, D. A. (2014). Optimising employee mental health: the relationship between intrinsic need satisfaction, job crafting, and employee well-being. Journal of Happiness Studies, 15(4), 957-977.
[17] Tian,Q.,T., \& Guan,H.,G. (2017). Work design revolution: research progress and prospect of job crafting. Human Resource Development of China. (3).

[18] Leana, C., Appelbaum, E., \& Shevchuk, I. (2009). Work process and quality of care in early childhood education: the role of job crafting. Academy of Management Journal, 52(6), 1169-1192. 